\title{
Modelling the 5-year cost effectiveness of tiotropium, salmeterol and ipratropium for the treatment of chronic obstructive pulmonary disease in Spain
}

\author{
Maureen P. M. H. Rutten-van Mölken • \\ Jan B. Oostenbrink · Marc Miravitlles • \\ Brigitta U. Monz
}

Received: 17 February 2006/ Accepted: 11 January 2007/Published online: 17 March 2007

(C) Springer-Verlag 2007

\begin{abstract}
Our objective was to assess the 5-year cost effectiveness of bronchodilator therapy with tiotropium, salmeterol or ipratropium for chronic obstructive pulmonary disease (COPD) from the perspective of the Spanish National Health System (NHS). A probabilistic Markov model was designed wherein patients moved between moderate, severe or very severe COPD and had the risk of exacerbation and death. Probabilities were derived from clinical trials. Spanish healthcare utilisation, costs and utilities were estimated for each COPD and exacerbation state. Outcomes were exacerbations, exacerbation-free months, qualityadjusted life years (QALYs), and cost(-effectiveness). The mean (SE) 5-year number of exacerbations was $3.50(0.14)$ for tiotropium, $4.16(0.40)$ for salmeterol and $4.71(0.54)$ for ipratropium. The mean (SE) number of QALYs was $3.15(0.08), 3.02(0.15)$ and 3.00 (0.20), respectively. Mean (SE) 5-year costs were $€ 6,424 \quad(€ 305)$ for tiotropium, $€ 5,869 \quad(€ 505)$ for
\end{abstract}

This study was financially supported by Boehringer Ingelheim International and Pfizer Global Pharmaceuticals.

M. P. M. H. Rutten-van Mölken ( $ه)$.

J. B. Oostenbrink

Institute for Medical Technology Assessment,

Erasmus MC, P.O. Box 1738,

3000 DR Rotterdam, The Netherlands

e-mail: m.rutten@bmg.eur.nl

M. Miravitlles

Servei de Pneumologia, Institut Clìnic del Tòrax

(IDIBAPS), Red Respira RTIC 03/11 ISCIII,

Hospital Clinic, Barcelona, Spain

B. U. Monz

Boehringer Ingelheim International,

Ingelheim am Rhein, Germany salmeterol, and $€ 5,181$ (€682) for ipratropium (2005 values). Ipratropium and tiotropium formed the costeffectiveness frontier, with tiotropium being preferred when willingness to pay (WTP) exceeded €639 per exacerbation-free month and $€ 8,157$ per QALY. In Spain, tiotropium demonstrated the highest expected net benefit for ratios of the willingness to pay per QALY, well within accepted limits.

Keywords Chronic obstructive pulmonary disease (COPD) - Bronchodilators · Model · Cost effectiveness - Quality-adjusted life year (QALY) . Spain

\begin{tabular}{|c|c|}
\hline Abbreviations & \\
\hline CE-plane & Cost-effectiveness plane \\
\hline COPD & $\begin{array}{l}\text { Chronic obstructive pulmonary } \\
\text { disease }\end{array}$ \\
\hline ER & Emergency room \\
\hline EQ-5D & $\begin{array}{l}\text { EuroQol 5-dimension utility } \\
\text { measurement instrument }\end{array}$ \\
\hline $\mathrm{FEV}_{1} \%$ pred. & $\begin{array}{l}\text { Forced expiratory volume in } 1 \mathrm{~s} \text { as } \\
\text { percentage of the predicted value }\end{array}$ \\
\hline $\mathrm{ml}$ & Millilitres \\
\hline SE & Standard error \\
\hline QALY & Quality-adjusted life year \\
\hline UK & United Kingdom \\
\hline VAT & Value added tax \\
\hline
\end{tabular}

Introduction

Owing to the chronic progressing nature and increasing prevalence of chronic obstructive pulmonary disease (COPD), its treatment will undoubtedly increase pressure 
on the drug budgets of developed countries in the future. Several new and competing treatments for COPD have recently been introduced, or may soon become available [1]. Healthcare authorities will be forced to decide upon reimbursement of these medications and will require high-quality information about the costs and effectiveness of these new drugs. Pharmacoeconomic evaluations provide this information and may guide the positioning of new drugs in the treatment spectrum of COPD.

To date, most economic evaluations of pharmacotherapy for COPD have been conducted alongside randomised controlled trials [2-7]. Consequently, their time horizon has been restricted to the duration of these trials, which is usually 6 months to 1 year, although economic evaluations of inhaled bronchodilators and corticosteroids piggybacked to clinical trials with durations of only 12-16 weeks have been reported $[4,5]$. For reimbursement authorities and formulary decision makers, these short-term economic evaluations are of limited value, because COPD requires long-term maintenance treatment on a daily basis. Furthermore, these policy makers will need results that represent their national or regional setting. Because it is not feasible to conduct long-term empirical costeffectiveness studies in all potential markets, some sort of modelling is required. This will allow expansion of the time horizon beyond that of a clinical trial, as well as the adapting and transferring of results from one setting to another. Decision analytical modelling has become an accepted approach to economic evaluations, and the use of modelling is supported by nearly all pharmaco-economic guidelines issued by health authorities involved in decision making about pricing and reimbursement of new pharmaceuticals, including Spain [8-11].

This study investigates the cost effectiveness of three bronchodilators for the maintenance treatment of COPD in Spain: tiotropium, salmeterol and ipratropium. Bronchodilators are central to the symptomatic management of COPD. Bronchodilators include three classes of medications: (1) the inhaled beta2-agonists, (2) the inhaled anticholinergics and (3) the oral methylxanthines. All three classes include drugs that are short acting or long acting. The most recently launched bronchodilator is tiotropium, an anticholinergic that provides $24 \mathrm{~h}$ bronchodilation with oncedaily dosing. It was developed as a more effective and more convenient alternative to ipratropium, the most frequently used short-acting anticholinergic bronchodilator in COPD, which has to be used four times daily. At the time tiotropium became available, salmeterol, which is used twice daily, was the most effective and the most frequently used long-acting bronchodilator from the beta2-agonists class. Orally administered methylxanthines, such as theophylline, are not recommended for routine use due to their unfavourable risk/benefit ratios. In a series of multinational trials tiotropium was directly compared with either placebo [12], ipratropium [13] or salmeterol [14]. The above-mentioned clinical trials have shown that tiotropium provides sustained bronchodilation, reduces exacerbation rate, and improves dyspnoea and healthrelated quality of life when compared with placebo or ipratropium. Improvements in lung function have been shown to be significantly better with tiotropium than with salmeterol [14]. Because information on the longterm cost effectiveness of these bronchodilators is lacking, a decision analytical model was developed to determine the 5-year cost effectiveness of tiotropium, salmeterol and ipratropium for the treatment of COPD. Efficacy data were derived from the above-mentioned clinical trials, and the potential cost effectiveness of inhaled bronchodilator therapy was assessed in scenario analyses. The model was populated with Spanish epidemiological data on COPD, Spanish healthcare utilisation, and unit costs and utilities based on Spanish population values. Spain was chosen because cost-effectiveness data on bronchodilator therapy from a Southern European country were lacking.

\section{Methods}

\section{The model}

This 5-year model can be characterised as a Markov model [15]. It builds on our earlier work in which we constructed a fully probabilistic Markov model to assess the 1-year cost effectiveness of tiotropium, salmeterol and ipratropium [16, 17]. All mathematical and technical details of this 1-year model have been published elsewhere [17]. In brief, all COPD patients were classified into three disease states of increasing severity, based on their pre-bronchodilator forced expiratory volume in $1 \mathrm{~s}\left(\mathrm{FEV}_{1}\right)$ : moderate COPD $\left(50 \% \leq\right.$ the percentage of the predicted value $\left(\mathrm{FEV}_{1} \%\right.$ pred. $)<80 \%)$, severe COPD $\left(30 \% \leq \mathrm{FEV}_{1} \%\right.$ pred. $<50 \%)$ and very severe COPD $\left(\mathrm{FEV}_{1} \%\right.$ pred. $\left.<30 \%\right)$. In pre-specified time intervals (Markov cycles) patients move between disease states and are at risk of experiencing an exacerbation, either severe or non-severe. All probabilities to move between disease states (transition probabilities) and to experience exacerbations that went into the 1-year model were obtained directly from six clinical trials comparing tiotropium 
with salmeterol, ipratropium or placebo (study codes: 205.114/205.117, 205.115/205.128, 205.122A/205.126A, 205.122B/205.126B, 205.130 and 205.137) [12-14]. Transition probabilities and exacerbation probabilities for tiotropium were based on the pooled patient-level data from all six clinical trials. To obtain the transition and exacerbation probabilities for the comparator arms, we applied the relative difference between tiotropium and the comparators in the individual trials to the probabilities of tiotropium that were derived from the pooled data [17].

All trials used similar inclusion and exclusion criteria. They enrolled patients with stable moderateto-severe COPD who had an $\mathrm{FEV}_{1}<65 \%$ (salmeterolcontrolled trials $<60 \%$ ) of predicted normal and $\mathrm{FEV}_{1} \leq 70 \%$ of forced vital capacity. The duration of these trials was 1 year for the those that compared tiotropium with placebo and ipratropium and 6 months for the trials that compared tiotropium with salmeterol. In total, 1,308 patients were randomly allocated to receive tiotropium, and 771 patients, 405 patients, and 179 patients were randomly selected to receive placebo, salmeterol and ipratropium. In all trials the same definitions of an exacerbation and its severity were used. An exacerbation was defined as a new onset or worsening of more than one symptom, such as cough, sputum, dyspnoea or wheeze, lasting for at least 3 days. Exacerbations were distinguished into severe and nonsevere exacerbations. A severe exacerbation was defined as 'incapacitating or inability to do work or usual activity'. All other exacerbations were non-severe. Severity was assessed by the clinical investigator. The risk of experiencing an exacerbation varied by disease state and treatment group. Given treatment group and disease state, exacerbation probabilities were assumed to be constant over time [17].

The current model expanded the time horizon from 1 year to 5 years and aimed to assess the cost effectiveness of tiotropium, salmeterol and ipratropium for treating COPD patients in Spain. To reflect the progressive nature of COPD in the long run and to incorporate mortality, an additional 'death state' was added to the model. The base-case analysis was performed from the perspective of the Spanish National Health Service (NHS) and included all costs covered by the NHS budget in euros at the 2005 value. A graphical presentation of the 5-year model is given in Fig. 1. In addition, an analysis from the societal perspective was performed. The difference between the societal perspective and the NHS perspective was that the first additionally included patients' co-payments for medications and the costs of lost production from day 1 of sick leave onwards; however, it excluded value added tax (VAT), as this does not represent a cost to society.

\section{Scenarios}

The first year of the model incorporated the benefits of therapy as observed in the clinical studies for improvement in lung function, which delayed the progression to subsequent COPD severity states [17]. The first year of the model also incorporated the reduction in the number of exacerbations that were found in the clinical trials [17]. These first year probabilities are shown in the upper part of Table 1 and in Table 2. Because the clinical trials did not provide data on the probabilities to move between disease states and to experience exacerbations after the first year, the 5 -year model was used to run three scenarios with different assumptions on transition and exacerbation probabilities for years 2 to 5 :

1. In the base-case scenario, the decline in $\mathrm{FEV}_{1}$ after the first year was assumed to be $52 \mathrm{ml} /$ year [18] in all treatment groups, whereas the exacerbation probabilities remained as they were for the first year. The decline of $52 \mathrm{ml}$ was the mean annual change in $\mathrm{FEV}_{1}$ among smokers and ex-smokers in the Lung Health Study [18]. The lower part of Table 1 shows the transition probabilities in the base-case scenario during years 2 to 5 , whereas Table 2 shows the exacerbation probabilities.

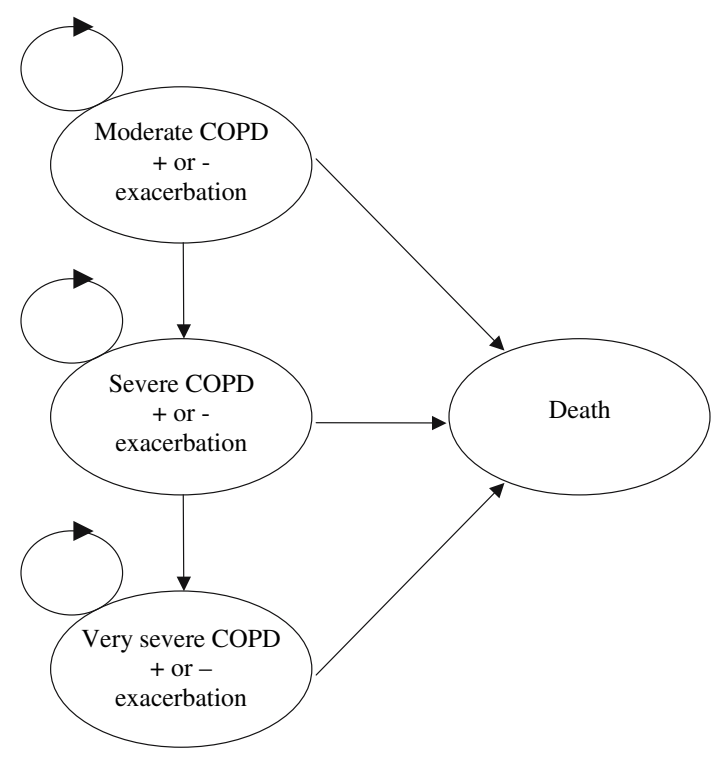

Fig. 1 Graphical presentation of the Markov model. Backward transitions, i.e. from very severe to severe COPD and from severe to moderate COPD, were allowed during the first year, but not thereafter. The circles on the top left of each COPD severity state indicate that the patients can stay in the same state 

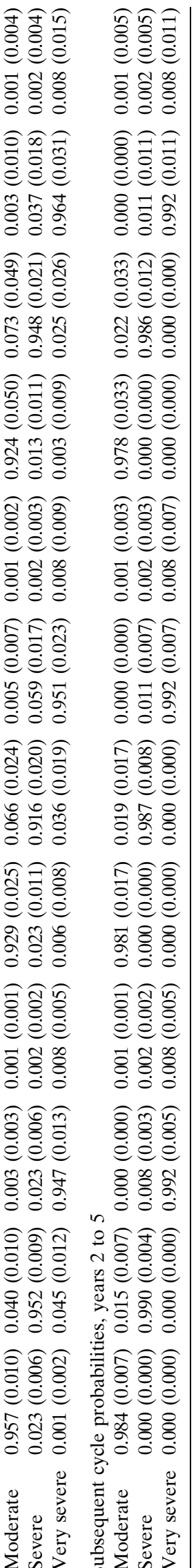

2. In the second scenario, both transition and exacerbation probabilities of the first year were assumed to remain constant during years 2 to 5 . In other words, the first year probabilities as shown in Table 1 (under the heading "Subsequent cycle probabilities, year 1") and 2 were applied to the later years.

3. In the third scenario, it was assumed that disease progression and exacerbation risk no longer differed between treatment groups after the first year. Exacerbation probabilities for tiotropium, salmeterol and ipratropium were assumed to be equal to the first year probabilities of the ipratropium group (columns 4 and 7 of Table 2). The assumption on lung function decline was the same as in the basecase scenario.

Note that the three scenarios do not differ with respect to the first year. During the first year, both forward transitions (transitions to worse disease states) and backward transitions (transitions to better disease states) are possible. In the base-case and third scenario, backward transitions during years 2 to 5 were not allowed, reflecting the progressive nature of COPD in general. Further note that no differences between treatments in terms of mortality risk were assumed.

Input data: baseline distribution of patients among disease states

The baseline distribution of patients among the disease states was based on a Spanish study by Miravitlles et al. [19] Re-analysis of the data according to the severity definition used in the model showed that, of 436 COPD patients, $55.2 \%$ had moderate disease, $34.9 \%$ had severe disease and $9.9 \%$ had very severe disease. A disease state for mild COPD was not included in the model, because mild COPD patients did not participate in the tiotropium trials.

Input data: death state

Probabilities of dying were based on all-cause mortality rates among Spanish patients with severe or very severe COPD as published by Miravitlles et al. [20]. The data from this study were re-analysed using the same cut-off values for the $\mathrm{FEV}_{1} \%$ predicted of severe and very severe COPD that were used in the model. The 1-year all-cause mortality rate was found to be 25 per 1,000 among patients with severe COPD [20], and the relative mortality risk in patients with very severe COPD compared with severe COPD was found to be 4.96 for a mean decline in $\mathrm{FEV}_{1} \%$ predicted of $19.4 \%$. 
Table 2 Mean (SE) exacerbation probabilities in the base-case scenario. (These probabilities were also used in scenario 2 . In scenario 3 the probabilities of ipratropium were also applied to tiotropium and salmeterol during years 2 to 5)

Probability of experiencing an exacerbation

\begin{tabular}{|c|c|c|c|c|c|c|}
\hline & & & & & & \\
\hline & Tiotropium & Salmeterol & Ipratropium & Tiotropium & Salmeterol & Ipratropium \\
\hline Moderate & $0.051(0.004)$ & $0.057(0.013)$ & $0.080(0.020)$ & $0.097(0.024)$ & $0.030(0.031)$ & $0.267(0.114)$ \\
\hline Severe & $0.075(0.003)$ & $0.089(0.011)$ & $0.097(0.013)$ & $0.136(0.018)$ & $0.138(0.033)$ & $0.188(0.041)$ \\
\hline Very severe & $0.096(0.005)$ & $0.104(0.016)$ & $0.102(0.022)$ & $0.192(0.027)$ & $0.207(0.048)$ & $0.186(0.062)$ \\
\hline
\end{tabular}

Under the assumption of constant proportional hazards, the relative mortality risk of patients in the trials was estimated to be 3.754 for very severe COPD and 0.248 for moderate COPD compared with severe COPD. We applied these relative risks to the all-cause mortality rate of 25 per 1,000 among patients with severe COPD to derive the mortality rates and, subsequently, the probabilities of dying, among patients with moderate and very severe COPD. These probabilities were the same for all treatment groups.

Input data: utilities

To reflect the impaired quality of life of patients with COPD, we attached utility weights to each disease severity state. These utilities were obtained from the EQ-5D scores at baseline in a subset of patients randomly accepted into the UPLIFT trial $(n=1,133)$ [21]. These scores were valued using the Spanish tariff [22]. A value of 1 represents perfect health, whereas 0 represents death. Mean (SE) utilities at baseline of the UPLIFT trial were calculated to be $0.809(0.008)$ in patients with moderate disease, $0.762(0.009)$ in patients with severe disease and 0.655 (0.024) in patients with very severe disease. During the months in which patients experienced an exacerbation, the utility value was reduced by $15 \%$ in the case of a nonsevere exacerbation [23] and by $50 \%$ in the case of a severe exacerbation [24]. Each year, the number of quality-adjusted life-years (QALYs) was calculated as the sum of the multiplications of the number of months in a particular disease state and the utility of that disease state, divided by 12 .

Input data: costs

The details and results of the cost estimates are presented in Table 3. Estimates of healthcare utilisation were primarily derived from two studies performed in Spain that had measured resource use and costs of the treatment of patients with COPD [25, 26]. Data from these studies were re-analysed to estimate resource use and costs by disease severity and exacerbation severity, using similar definitions as applied in the model. In the model, healthcare utilisation associated with COPD maintenance therapy varied by disease severity. Healthcare utilisation during exacerbations varied by the severity of the exacerbation (severe or non-severe). Calculations were conservative in assuming that costs per disease severity state and costs per severe or nonsevere exacerbation were equal across treatment groups. Resources for maintenance therapy included visits to respiratory physicians inside and outside the hospital, visits to the general practitioner, pulmonary function tests, blood tests, imaging tests and respiratory medications. Resources associated with a non-severe exacerbation included general practitioner and respiratory physician visits and medications. Besides physician visits and outpatient medications, resources associated with severe exacerbations also included hospital admissions and visits to the emergency room (ER) department. Costs of pulmonary function and other tests, as well as costs of medications that were prescribed during inpatient stay or during ER visits, were not measured separately, because they were included in the overall costs per in-patient day and the costs per ER visit.

Unit costs of healthcare resources were obtained from the SOIKOS health database and have been described in detail elsewhere [25]. They were updated to 2005 using the Spanish General Consumer Price Index. Acquisition costs of pulmonary drugs from the NHS perspective were based on public prices and calculated as the ex-factory prices multiplied by a mark-up of 1.596 to convert these prices to public prices [27]. The co-payment of $10 \%$ of the public price for the people in the work force was excluded. It was estimated that $33.2 \%$ of Spanish COPD patients had to pay these copayments because they were still in the work force [27]. From a societal perspective, the drug costs included copayment but excluded the $4 \%$ VAT that is included in the public prices. Costs of tiotropium, salmeterol and ipratropium from the NHS perspective were $€ 1.80$, $€ 1.20$ and $€ 0.19$ per day, respectively. From the soci- 
etal perspective these costs were $€ 1.76$ for tiotropium, $€ 1.18$ for salmeterol and $€ 0.18$ for ipratropium.

The NHS covers the costs of absence from work due to illness from the 16th day of sick leave onwards. The NHS pays $60 \%$ of gross salary during days 16 to 20 and $75 \%$ from day 21 onwards. When we were calculating costs from the NHS perspective, these costs were added to the costs of all severe exacerbations with sick leave longer than 15 days (approximately $7 \%$ of all severe exacerbations). The costs from the societal perspective included the costs of lost production during all days of absence from paid work.

The resulting mean (SE) annual costs of maintenance therapy from an NHS perspective were $€ 430$ $(€ 24)$ for a patient with moderate COPD, $€ 587(€ 34)$ for a patient with severe COPD and $€ 818(€ 58)$ for a patient with very severe COPD. The corresponding values from the societal perspective were $€ 429$ (€21), $€ 586(€ 34)$ and $€ 816$ (€58). The mean (SE) costs of exacerbations from the NHS perspective were
Table 3 Mean healthcare utilisation $(H U)$, unit costs, and mean (SE) total costs of maintenance therapy and exacerbations from the NHS perspective for Spain in euros at the 2005 value $(H U$ healthcare utilisation, $R P$ respiratory physician, $G P$ general practitioner, $I C U$ intensive care unit, exa exacerbation)

\begin{tabular}{|c|c|c|c|c|c|c|c|c|c|c|c|c|}
\hline \multirow[t]{2}{*}{ Maintenance therapy } & \multirow{2}{*}{\multicolumn{2}{|c|}{$\begin{array}{l}\text { Unit } \\
\text { costs }\end{array}$}} & \multicolumn{4}{|c|}{ Moderate COPD } & \multicolumn{4}{|c|}{ Severe COPD } & \multicolumn{2}{|c|}{ Very severe COPD } \\
\hline & & & \multicolumn{2}{|c|}{$\begin{array}{l}\text { HU per patient } \\
\text { per year }\end{array}$} & \multicolumn{2}{|c|}{$\begin{array}{l}\text { Total } \\
\text { costs }\end{array}$} & \multicolumn{2}{|c|}{$\begin{array}{l}\text { HU per patient } \\
\text { per year }\end{array}$} & \multicolumn{2}{|c|}{$\begin{array}{l}\text { Total } \\
\text { costs }\end{array}$} & $\begin{array}{l}\text { HU per patient } \\
\text { per year }\end{array}$ & $\begin{array}{l}\text { Total } \\
\text { costs }\end{array}$ \\
\hline \multirow{3}{*}{$\begin{array}{l}\text { Visit RP in hospital } \\
\text { Visit RP outside hospital } \\
\text { Visit GP }\end{array}$} & \multicolumn{2}{|c|}{79.85} & \multicolumn{2}{|l|}{0.27} & \multicolumn{2}{|c|}{$22(1)$} & \multicolumn{2}{|l|}{0.44} & \multicolumn{2}{|c|}{$35(2)$} & 0.63 & $50(3)$ \\
\hline & \multicolumn{2}{|c|}{23.77} & \multicolumn{2}{|l|}{0.38} & \multicolumn{2}{|c|}{$9(1)$} & \multicolumn{2}{|l|}{0.57} & \multicolumn{2}{|c|}{$14(1)$} & 0.65 & $15(1)$ \\
\hline & \multicolumn{2}{|c|}{8.35} & \multicolumn{2}{|l|}{2.59} & \multicolumn{2}{|c|}{$22(1)$} & 2.88 & & \multicolumn{2}{|c|}{$24(1)$} & 3.27 & $27(1)$ \\
\hline Thorax X-ray & \multicolumn{2}{|c|}{19.67} & 1.03 & & & & 1.18 & & 23 & & 1.49 & $29(1)$ \\
\hline ECG & 22. & & 0.80 & & & & 0.87 & & 20 & & 1.15 & $26(1)$ \\
\hline Spirometry & 40 . & & 0.55 & & & & 0.66 & & 27 & & 0.92 & $37(2)$ \\
\hline Blood analyses & 19. & & 1.41 & & & & 1.43 & & 28 & & 1.58 & $31(1)$ \\
\hline Blood gases & 27. & & 0.33 & & & 1) & 0.56 & & 15 & & 0.67 & $18(1)$ \\
\hline Influenza vaccination & 5.4 & & 0.48 & & & 1) & 0.64 & & $4(1$ & & 0.72 & $4(1)$ \\
\hline Theophylline & 0.1 & & 122.06 & & & & 161.77 & & 30 & & 159.07 & $29(5)$ \\
\hline Mucolytics & 0.3 & & 39.74 & & & & 48.31 & & 14 & & 80.60 & $24(4)$ \\
\hline Oral corticosteroids & 0.2 & & 21.54 & & & 3) & 23.73 & & $7(1$ & & 78.48 & $23(6)$ \\
\hline Inhaled corticosteroids & 0.6 & & 224.84 & & 138 & & 224.84 & & 138 & (15) & 292.00 & $179(22)$ \\
\hline Oxygen & 3.7 & & 21.32 & & 79 & & 44.26 & & 165 & (30) & 77.87 & $290(52)$ \\
\hline Other & & & & & & & & & 44 & & & $34(5)$ \\
\hline Total costs per patient per & & & & & 430 & & & & 587 & (34) & & $818(58)$ \\
\hline Exacerbations & Unit & & n-severe e & acerbat & ion & Sev & ere exace & bation & & Stud & medication costs & per day \\
\hline & & & J per exa & Total & costs & $\mathrm{HL}$ & per exa & Total c & & & & \\
\hline $\mathrm{ICU}^{\mathrm{a}}$ & 1,284 & - & & _- & & 0.2 & & $374(2$ & & Tio & pium & .80 \\
\hline Non-ICU ${ }^{\mathrm{a}}$ & 368 & - & & - & & 4.1 & & $1,529($ & 307) & Saln & terol & .20 \\
\hline Emergency room & 115 & - & & - & & 0.9 & & $108(11$ & & Ipra & ropium & 19 \\
\hline Visit GP & 8.35 & & 64 & $14(1)$ & & 1.0 & & $8(3)$ & & & & \\
\hline Visit RP in hospital & 79.85 & - & & 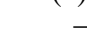 & & 0.5 & & $42(10)$ & & & & \\
\hline Antibiotics $^{\mathrm{b}}$ & 5.00 & 11 & & $55(7)$ & & 7.5 & & $38(7)$ & & & & \\
\hline Oral corticosteroids ${ }^{\mathrm{b}}$ & 0.29 & & 69 & $1(1)$ & & 4.9 & & $1(1)$ & & & & \\
\hline Inhaled corticosteroids ${ }^{\mathrm{b}}$ & 0.61 & & 01 & $4(1)$ & & 3.7 & & $2(1)$ & & & & \\
\hline Oxygen $^{\mathrm{b}}$ & 3.73 & & 05 & $8(3)$ & & 5.3 & & $20(2)$ & & & & \\
\hline Other & & & & $1(1)$ & & & & $1(1)$ & & & & \\
\hline NHS sick leave benefit ${ }^{c}$ & 74.09 & & & & & & & $13(2)$ & & & & \\
\hline Total costs per exacerbatio & & & & $83(7)$ & & & & 2,136 & 125) & & & \\
\hline
\end{tabular}

For medications and oxygen $\mathrm{HU}$ is expressed in number of days during which the medication or oxygen was used

${ }^{a}$ Of all severe exacerbations $52 \%$ required hospital admission. Of those, $14 \%$ were to the ICU. The length of stay on ICU is 4 days, and the length of stay on non-ICU is 8 days. Hence, $0.52 \times 0.14 \times 4=0.29$ and $0.52 \times 8=4.16$

${ }^{\mathrm{b}}$ Only medications prescribed in ambulatory settings. Costs of medications administered in hospital and at emergency rooms are included in costs of hospital stay/emergency room visit

c Average labour costs per day in Spain are €74.09. Of Spanish COPD patients, 33.2\% have paid employment; $0.332 \times$ $€ 74.09=€ 24.60$. The NHS covers $60 \%$ of these labour costs during days $16-20$ of the sick leave episode and $75 \%$ from day 21 onwards. Of severe exacerbations, $7 \%$ are associated with sick leave longer than 15 days. The duration of these absence spells is 26 days. So, $(5 \times 0.6 \times € 24.60+6 \times 0.75 \times € 24.60) \times 0.07=€ 13.43$ 
$€ 83(€ 7)$ for a non-severe exacerbation and $€ 2,176$ $(€ 425)$ for a severe exacerbation. From a societal perspective these costs were $€ 121$ (€7) and $€ 3,912$ (€543), respectively.

\section{COPD severity}

To investigate the impact of COPD severity on the cost effectiveness of the bronchodilators we ran sensitivity analyses with the base-case scenario, assuming that, at the start of the model, $100 \%$ of the patients had either moderate, severe or very severe COPD.

\section{Discounting}

Because of time preference, costs and effects that will arise in the future are usually valued lower than costs and effects in the present. Discounting is used to calculate the present value of future costs and effects. In accordance with proposed Spanish guidelines, an annual discount rate of $6 \%$ has been adopted [28]. In the base-case analysis, costs and health outcomes were discounted at the same rate, whereas, in sensitivity analyses, discount rates of $0 \%$ for both costs and effects, and $6 \%$ for costs combined with $3 \%$ for effects, were applied. In scenarios 2 and 3 the same $6 \%$ discount rate was used as in the base-case scenario.

\section{Probabilistic sensitivity analysis}

To facilitate the interpretation of results of economic analyses, the reporting of uncertainty associated with costs and outcomes is equally important as reporting the point estimates of these parameters. Hence, the model was designed fully probabilistic, and uncertainty around the probabilities to move between disease states, the probabilities to experience exacerbations, utilities and healthcare utilisation was addressed by defining a probability distribution for each input parameter [17]. The uncertainty around these input parameters was propagated through the model simultaneously by conducting second-order Monte Carlo simulations. This means making random draws of the uncertain parameters from their probability distribution, running the model for each set of parameters that is drawn, and collecting the outputs from each run [29]. The current results were based on 5,000 iterations.

The main outcome measures of the model were mean and standard error (SE, being the standard deviation of the 5,000 iterations) of the 5-year costs per patient, exacerbation-free months and quality-adjusted life years. The presentation of incremental cost effec- tiveness was based on the hierarchy of outcomes, i.e. each treatment option was compared with the next best treatment option in terms of effectiveness. The uncertainty around costs and effects was further explored by plotting of the 5,000 iterations on incremental cost-effectiveness planes (CE-planes) [30] and by presenting cost-effectiveness acceptability curves $[30,31]$ and frontiers [32]. The acceptability curve presents the probability that a treatment is the most cost effective of the three treatments at different threshold values for cost effectiveness (ceiling ratio), whereas the cost-effectiveness acceptability frontier demonstrates which of the three treatments should be adopted because it results in the highest expected net benefit, given the ceiling ratio. The net benefit is calculated as the total costs $(C)$ minus the effects $(E)$ multiplied by the ceiling ratio $(C-(E \times$ ceiling ratio $))$ [33]. The model was programmed in Microsoft EXCEL.

\section{Results}

\section{Health outcomes}

The results of the Markov simulation for the different scenarios are presented in Table 4. In the base-case scenario, the mean (SE) number of exacerbations in 5 years was $3.50(0.14)$ in the tiotropium group, 4.16 $(0.40)$ in the salmeterol group and $4.71(0.54)$ in the ipratropium group. The corresponding mean (SE) number of exacerbation-free months was 46.83 (1.11) in the tiotropium group, 45.29 (2.12) in the salmeterol group and 44.89 (2.86) in the ipratropium group. Estimates of the number (SE) of QALYs were $3.15(0.08)$, $3.02(0.15)$ and $3.00(0.20)$, respectively. In all scenarios, differences in exacerbation-free months and QALYs between treatment groups were consistently in favour of the tiotropium group. Applying the first year probabilities to all subsequent years (scenario 2 ) increased the difference in exacerbation-free months between tiotropium and the other treatment groups to approximately 2.0 and the difference in QALYs to approximately 0.19. Assuming similar exacerbation probabilities across treatment groups in year 2 to 5 (scenario 3) reduced the differences between treatment groups considerably. In this analysis, the difference in exacerbation-free months between tiotropium and the other treatment groups was just above 1, and the difference in QALYs was about 0.13. There was almost no difference in health outcomes between ipratropium and salmeterol in this scenario. 
Table 4 Results of the Markov simulation for the base-case analysis from the NHS perspective covering a time period of 5 years. The table gives mean (SE) or mean $(95 \%$ confidence interval). $Q A L Y$ quality-adjusted life year, Tio tiotropium, Salm salmeterol, Ipra Ipratropium, exa exacerbations, CE-ratio costeffectiveness ratio, exa-free exacerbation-free

\begin{tabular}{lccc}
\hline Outcomes base-case analysis (cumulative over 5 years) & & \\
\hline Health outcome & Tiotropium & Salmeterol & Ipratropium \\
Exacerbations & $3.50(0.14)$ & $4.16(0.40)$ & $4.71(0.54)$ \\
Exacerbation-free months & $46.83(1.11)$ & $45.29(2.12)$ & $44.89(2.86)$ \\
Life-years & $4.19(0.10)$ & $4.12(0.19)$ & $4.13(0.26)$ \\
QALYs & $3.15(0.08)$ & $3.02(0.15)$ & $3.00(0.20)$ \\
& & & \\
Costs in 2005 Euro mean (SE) & & & \\
Costs of exacerbations & $1289(231)$ & $1558(374)$ & $2335(584)$ \\
Costs of maintenance therapy & $2380(114)$ & $2514(183)$ & $2558(238)$ \\
Costs of study medications & $2757(65)$ & $1801(83)$ & $279(18)$ \\
Total costs & $6424(305)$ & $5869(505)$ & $5181(682)$
\end{tabular}

\section{Differences and incremental cost-effectiveness ratios per scenario}

\section{Base-case scenario}

Differences in:

Exacerbation-free months

Tio versus salm

QALYs

$1.54(-2.50 ; 6.81)$

Costs

$0.14(-0.16 ; 0.49)$

$555(-647 ; 1651)$

Incremental CE-ratios

Cost per exa-free month

Quadrant distribution

CE-plane exa-free month

Cost per QALY

Quadrant distribution

CE-plane QALY

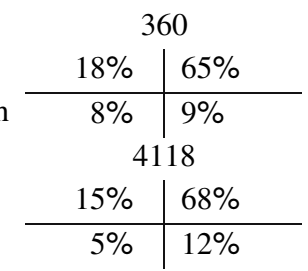

\section{Scenario 2}

Differences in:

Tio versus salm

$1.93(-2.58 ; 8.62)$

$0.19(-0.12 ; 0.63)$

$418(-869 ; 1692)$

217

2239

Tio versus Salm

1.07 (-3.02; 6.46)

$0.13(-0.16 ; 0.49)$

$831(-860 ; 2489)$

777

6446
Salm versus Ipra

0.40 (-6.26; 7.99)

$0.02(-0.43 ; 0.53)$

$688(-1012 ; 2291)$

1711

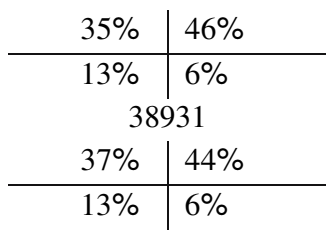

Salm versus Ipra

0.21 (-7.86; 9.77)

$0.002(-0.54 ; 0.63)$

$758(-1151 ; 2541)$

3698

348971

Salm versus Ipra

-0.008 (-6.54; 8.00)

$0.001(-0.45 ; 0.55)$

$1205(-648 ; 3066)$

$-144075$

690861

\section{Costs}

In the base-case scenario from the NHS perspective, mean (SE) total costs over 5 years were lowest in the ipratropium group, with $€ 5,181$ (€682) per patient (Table 4). Costs were $€ 5,869$ (€505) in the salmeterol group and $€ 6,424$ (€305) in the tiotropium group. The higher costs in the tiotropium and salmeterol 
group were largely due to the higher costs of study medication. Compared with those of ipratropium, the 5 -year costs of study medication were $€ 2,477$ ( $€ 67$ ) higher in the tiotropium group and $€ 1,521$ (€85) higher in the salmeterol group. Savings in other categories of costs, mainly exacerbation-related costs, offset approximately half of these additional study medication costs.

Because of a smaller difference in exacerbation rate between treatment groups in scenario 3, the difference in total costs between ipratropium on the one hand and tiotropium and salmeterol on the other hand increased by $64 \%$ and $75 \%$, respectively. The impact of applying the first-year transition probabilities to years 2 to 5 (scenario 2) on the 5-year differences in costs between treatment groups was much less (Table 4).
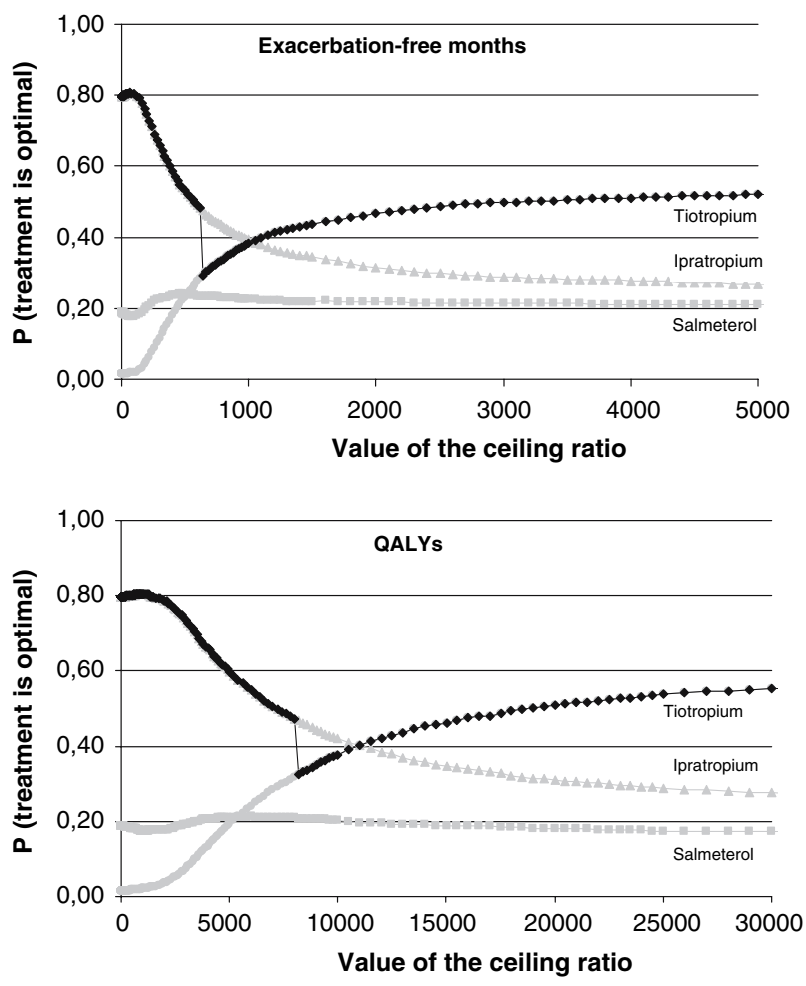

Fig. 2 Cost-effectiveness acceptability curves and frontier of the costs per exacerbation-free month and the cost per QALY. Ceiling ratio: threshold value for the cost-effectiveness ratio in euros. The curves in grey represent the probability that a treatment is cost effective for a given value of the ceiling ratio. In the case of skewed distributions of the ratio, the treatment with the highest probability of being cost effective is not always the treatment with the highest expected net benefit. The acceptability frontier (in black) shows which treatment is associated with the highest expected net benefit for each value of the ceiling ratio
Cost effectiveness

In the base-case scenario from the NHS perspective estimates of the incremental costs per exacerbationfree month were $€ 360$, when tiotropium was compared with salmeterol, and $€ 1,711$ when salmeterol was compared with ipratropium. The corresponding incremental costs per QALY were $€ 4,118$ and $€ 38,931$, respectively. The distribution of the results of the 5,000 model iterations on the CE-planes is also reported in Table 4. The comparisons between tiotropium and salmeterol show that the majority of simulations (approximately 65\%) lie in the upper-right quadrants, signifying better health outcomes and higher costs for tiotropium. The CE-planes comparing salmeterol with ipratropium show that about $35 \%$ of the dots are found in the upper-left and about $45 \%$ in the upper-right quadrant, signifying similar health outcomes and higher costs for salmeterol.

Figure 2 shows the cost-effectiveness acceptability curves in the base-case scenario from the NHS perspective. The curves show that, in terms of exacerbation-free months, ipratropium has the highest probability of being cost effective when the threshold value for cost per additional exacerbation-free month is below $€ 1,050$. In terms of QALYs, ipratropium has the highest probability of being the most cost effective for all threshold values for cost per QALY below $€ 11,000$. Above these values, tiotropium has the highest probability of being cost effective. However, when the distribution of the cost-effectiveness ratio is skewed, as is the case in the presented analyses, the treatment with the highest probability of being cost effective is not always the treatment with the highest expected net benefit [32]. In Fig. 2, the cost-effectiveness acceptability frontier is drawn in bold. The frontier follows the curve of the treatment with the highest expected net benefit for a given value of the costeffectiveness threshold. Hence, it indicates which treatment should be chosen because it is optimal. The bold curve demonstrates that both ipratropium and tiotropium are on the frontier. For any ceiling ratio above $€ 639$ per exacerbation-free month and above $€ 8,157$ per QALY, tiotropium is the preferred treatment option. In scenario 2 , these threshold values were lower: $€ 551$ and $€ 6,226$, respectively, whereas the corresponding values in scenario 3 were $€ 1,918$ and $€ 15,635$. The point on the frontier where the most optimal treatment switches from ipratropium to tiotropium in Fig. 2 corresponds to the base-case incremental cost-effectiveness ratio for the comparison between these two bronchodilators. Figure 2 also shows that, although tiotropium has the highest prob- 
ability of being the most optimal treatment, this probability is $58 \%$ at maximum. This $58 \%$ is the value at which the acceptability curve of tiotropium using QALYs becomes asymptotic.

\section{Societal perspective}

From the societal perspective, overall mean (SE) costs increased in all treatment groups to $€ 6,574$ (€321) for tiotropium, $€ 6,125$ ( $€ 541)$ for salmeterol and $€ 5,545$ $(€ 720)$ for ipratropium. However, the savings in exacerbation costs generated by tiotropium and salmeterol compared with ipratropium were higher from a societal perspective than from the NHS perspective. Consequently, from the societal perspective, the difference in overall costs between the treatment groups was reduced. Also, the incremental costs per exacerbation-free month were reduced to $€ 308$, for the comparison tiotropium versus salmeterol, and $€ 1,375$ for the comparison salmeterol versus ipratropium. The incremental cost per QALY were reduced to $€ 3,483$, for the comparison tiotropium versus salmeterol, and $€ 35,158$ for the comparison salmeterol versus ipratropium. Tiotropium had the highest expected net benefit for any value of the cost-effectiveness threshold above $€ 547$ per exacerbation-free month and above $€ 7,076$ per QALY. Below these values ipratropium is preferred.

\section{Impact of COPD severity}

When the model was run separately for patients with either moderate, severe or very severe COPD, it showed that the threshold value above which tiotropium had the highest expected net benefit increased with the severity of COPD. The threshold values for the costs per QALY above which tiotropium became the preferred option were $€ 7,600$ for moderate COPD, $€ 8,800$ for severe COPD and $€ 12,500$ for very severe COPD. Below these values ipratropium was preferred. Tiotropium had the highest expected net benefit when the ceiling ratios for cost per exacerbation free month were $€ 560$ for moderate COPD, $€ 700$ for severe COPD and $€ 1,200$ for very severe COPD. Below these values ipratropium had the highest expected net benefit.

\section{Discount rates}

Because discounting affects both health outcomes and costs, the effect of discounting in this study was small. Analyses based on discount rates of 3\% and $0 \%$ showed almost similar cost-effectiveness ratios. Discounting health outcomes at a lower rate than costs led to costeffectiveness ratios that were slightly more in favour of tiotropium.

\section{Discussion}

Bronchodilators form the main therapy for the symptomatic relief of respiratory symptoms in COPD patients. In this study we have constructed a decision analytical model to synthesise clinical trial data on the effectiveness of bronchodilator treatment. We have shown how scenario analyses can be used to extend the time horizon of the cost-effectiveness study beyond that of the clinical trials. In addition, we have shown how a model can be populated with country-specific data to obtain estimates of the cost-effectiveness of bronchodilators in the Spanish setting.

This comprehensive country adaptation and extension of the existing short-term Markov model [17] required much more than just the imputation of Spanish unit costs. We searched for Spanish sources for almost every type of input data, ranging from the distribution of COPD severity stages, mortality, the proportion of COPD patients in the work force, utilities, resource use and unit costs. We re-analysed patient-level data from previously published Spanish studies on prevalence, mortality, EQ-5D, and resource utilisation [19-21, 25, 26] to ensure that these Spanish data matched the model's definitions of COPD severity and exacerbation severity. This included obtaining point estimates as well as distributions of these input data. The strength of the Spanish data is also that mainly patients from general practices were included, thus reflecting the routine care setting closely. The majority of Spanish patients are treated by general practitioners, and the proportion of patients referred to specialist care by pulmonologists is smaller than in many other west European countries [34]. This may be related to the gate-keeping function of the general practitioner in Spain that is not enforced in some other European countries.

The base-case scenario showed that tiotropium was associated with an approximately $16 \%$ reduction in exacerbations when compared with salmeterol. Salmeterol was associated with $12 \%$ reduction in exacerbations, when compared with ipratropium. Differences between the three treatment groups in terms of QALYs were small. That was expected, given the 5-year time horizon and treatments that do not directly affect survival. The distribution of dots on the CE-planes showed that the higher effectiveness of salmeterol over ipratropium was associated with more 
uncertainty than the higher effectiveness of tiotropium over salmeterol, because, for the latter comparison, a larger proportion of the dots was found on the right side of the CE plane.

Beside effectiveness, the choice between bronchodilators must involve many considerations, including economic considerations of costs and cost effectiveness. The final result of this cost-effectiveness analysis is an acceptability frontier that demonstrated that tiotropium has the highest expected net benefit for a threshold value of the costs per QALY that is well within the limits of other adopted therapies in Spain [35] and in other countries like the UK [36]. This threshold value increases with the severity of COPD, because the differences between treatments in the probabilities of developing a (severe) exacerbation decrease as COPD severity increases. Nevertheless, the cost-effectiveness threshold in the most severely affected patients is still relatively low.

The threshold value for costs per additional exacerbation-free month of $€ 639$ above which tiotropium becomes the optimal choice in Spain is higher than the ceiling ratios above which tiotropium became most cost effective in the Netherlands $(€ 0)$ and Canada $(€ 10)$ in the 1-year model [17]. Similarly, when QALYs were used as the outcome measure, the ceiling ratio of $€ 8,157$ per QALY above which tiotropium is the preferred option in Spain is also higher than in The Netherlands and Canada, where tiotropium became the preferred option when decision makers could afford to pay more than $€ 0$ and $€ 120$ for a QALY, respectively. This difference between these countries is largely driven by the relatively low acquisition costs of ipratropium in Spain, which is related to the fact that there is no widespread use of the more expensive dry powder formulation of ipratropium. In addition, the savings due to the reduction in exacerbations by tiotropium and salmeterol are less, because the costs of treating a severe exacerbation in Spain are lower than in The Netherlands and Canada. This is caused by a smaller proportion of patients hospitalised, as well as a shorter length of stay. As a result, tiotropium generated very small net savings in the Netherlands and similar costs to the other two bronchodilators in Canada, whereas it increased total costs in Spain. Nevertheless, in the three countries investigated, the economic evaluations indicated that the health benefits gained with tiotropium are either at almost no additional costs or at costs that appear reasonable and acceptable, given other adopted treatments $[37,38]$.

Our base-case scenario was conservative with respect to lung function decline, as we assigned a similar decline of $52 \mathrm{ml} /$ year to the patients in all treatment groups after the first year. We chose this to be the best assumption for the base-case scenario, because there is little evidence in the literature that bronchodilators alter the rate of decline of lung function [39]. However, frequent exacerbations seem to accelerate the decline in lung function [40, 41]. Therefore, our base-case scenario might have underestimated the long-term effect of a bronchodilator that reduces the number and severity of exacerbations. For that reason, we developed the second scenario, which is the least conservative, because we assumed that the differences in lung function decline and exacerbation rates between the three treatments that were observed during the first year would remain during the 4 years thereafter. This scenario is most favourable for tiotropium, and we see the threshold value above which tiotropium becomes most cost effective drop to $€ 551$ per exacerbation-free month and $€ 6,226$ per QALY. In addition to this optimistic scenario, we developed a very conservative scenario by completely eliminating the difference in exacerbation rate between the treatment groups after the first year and assuming that exacerbation rates would resemble the rate observed in the ipratropium group. This third scenario also assumed a similar decline in lung function across treatment groups of $52 \mathrm{ml} /$ year. This third scenario is conservative because exacerbations were the main drivers of cost effectiveness. The threshold value above which tiotropium becomes most cost effective in this third scenario increases to $€ 1,918$ for an additional exacerbation-free month and $€ 15,635$ for a QALY, yet is still below acceptable limits reported for the Spanish setting. Below that, ipratropium was most cost effective. Altogether, these three scenarios give the range within which to expect the 5-year cost effectiveness of these bronchodilators. Note, that we had no scenarios assuming a differential effect of the treatments on, for example, utilities or costs of a single exacerbation, because the evidence of such effects is lacking. Furthermore, all scenarios had a time horizon of 5 years to meet the requirements of many European reimbursement authorities who prefer extensions to time horizons reflecting their budgeting process (typically for 35 years). Lifetime models that primarily aim to compare medications are soon outdated by the development of new therapeutic options.

When the cost-effectiveness analysis was conducted from a societal perspective, the threshold value above which tiotropium is the preferred option is reduced considerably to $€ 547$ per exacerbation-free month and $€ 7,076$ per QALY. This improved cost effectiveness is 
primarily related to increasing exacerbation costs, because of the inclusion of the costs of production losses. Hence, a reduced exacerbation rate is associated with higher savings. The NHS perspective also included sick leave benefits paid for by the NHS from day 16 onwards. However, this applies only to severe exacerbations where it was less than $1 \%$ of its costs. In contrast, from a societal perspective, costs of production losses are $10 \%$ of the costs of a severe exacerbation and $30 \%$ of the costs of a non-severe exacerbation.

We chose two outcome measures that are among the most relevant in the lives of patients with COPD: being free of exacerbations and having a good quality of life. The concept of an exacerbation-free month is comparable to the concept of a symptom-free day, which is a frequently used outcome measure in asthma [42]. As it is a positive outcome (i.e. more is better), its cost-effectiveness ratio is easier to interpret than the ratio 'cost per exacerbation avoided'. In general, the number of exacerbations is not a good outcome measure to use in long-term models, especially when treatment improves survival. This outcome measure will bias the treatment which improves survival, because patients can experience exacerbations during the life years gained. In contrast, the number of exacerbation-free months takes account of the fact that the added years of life are partly lived free of exacerbations.

The 1-year model, as well as the currently presented long-term model, has been used in reimbursement negotiations in several countries. The major strength of this model is its transparency. We have fully disclosed the model structure as well as all the input. Costs per exacerbation and costs per disease state do not differ between the three treatment groups, and mortality rates by disease severity were also set equally for all treatment groups. Hence, the difference in cost effectiveness is driven by the difference in acquisition costs of the study drugs and the difference in their effectiveness, i.e. the difference in the probabilities to move between disease states and the differences in exacerbation risks. For the first year of the model, estimates of effectiveness were directly obtained from patientlevel data from clinical trials. Hence, estimates of probabilities were not based on expert opinion or literature, as is often the case in modelling studies. We acknowledge that differences may exist between trial results from which we derived the model input and the effectiveness of therapy in routine daily practice. A model can be used to adapt the trial data to better represent daily practice. Particularly, compliance is known to be worse in daily practice. However, not accounting for compliance was conservative for tiotropium and salmeterol, since once-daily and twice-daily dosing is more likely to be accompanied by long-term compliance than is four-times daily dosing in chronic diseases [43]. Owing to the doubleblind, double-dummy design of the clinical trials, the dosing frequency was the same in the treatment groups, and any impact of dosing frequency on outcomes was eliminated.

In conclusion, our model has demonstrated that tiotropium is the treatment with the highest expected net benefit, if decision makers can afford to spend additional budget to gain additional health benefits. The threshold value of the costs per QALY at which tiotropium becomes the preferred treatment is well within acceptable limits (i.e. $€ 8,157$ from the NHS perspective and $€ 7,076$ from the societal perspective).

\section{References}

1. Barnes, P.J.: Chronic obstructive pulmonary disease: new treatments for COPD. Thorax 58(9), 803-808 (2003)

2. Ayres, J.G., Price, M.J., Efthimiou, J.: Cost-effectiveness of fluticasone propionate in the treatment of chronic obstructive pulmonary disease: a double-blind randomized, placebocontrolled trial. Respir. Med. 97(3), 212-220 (2003)

3. Gallefoss, F., Bakke, P.S.: Cost-benefit and cost-effectiveness analysis of self-management in patients with COPD-a 1-year follow-up randomized, controlled trial. Respir. Med. 96(6), 424-431 (2002)

4. Hogan, T.J., Geddes, R., Gonzalez, E.R.: An economic assessment of inhaled formoterol dry powder versus ipratropium bromide pressurized metered dose inhaler in the treatment of chronic obstructive pulmonary disease. Clin. Ther. 25(1), 285-297 (2003)

5. Jones, P.W., Wilson, K., Sondhi, S.: Cost-effectiveness of salmeterol in patients with chronic obstructive pulmonary disease: an economic evaluation. Respir. Med. 97(1), 20-26 (2003)

6. Rutten-van Mölken, M.P.M.H., van Doorslaer, E.K.A., Jansen, M.C.C., Kerstjens, H.A.M., Rutten, F.F.H.: Costs and effects of inhaled corticosteroids and bronchodilators in asthma and chronic obstructive pulmonary disease. Am. J. Respir. Crit. Care Med. 151, 975-982 (1995)

7. Torrance, G., Walker, V., Grossman, R., Mukherjee, J., Vaughan, D., La Forge, J., Lampron, N.: Economic evaluation of Ciprofloxacin compared with usual antibacterial care for the treatment of acute exacerbations of chronic bronchitis in patients followed for 1 year. PharmacoEconomics 16 (5 pt1), 499-520 (1999)

8. Gold, M.R., Siegel, J.E., Russell, L.B., Weinstein, M.C.: Cost-effectiveness in Health and Medicine, 1st edn. Oxford University Press, New York (1996)

9. Hjelmgren, J., Berggren, F., Andersson, F.: Health economic guidelines-similarities, differences and some implications. Value Health 4(3), 225-50 (2001)

10. International Society For Pharmacoeconomics \& Outcomes Research (ISPOR): Pharmacoeconomic guidelines around the world. http://www.ispor.org/PEguidelines/index.as. Accessed July 2005 
11. National Institute for Clinical Excellence: Guide to the Methods of Technology Appraisal. NICE, London (2004)

12. Casaburi, R., Mahler, D.A., Jones, P.W., Wanner, A., San, P.G., ZuWallack, R.L., Menjoge, S.S., Serby, C.W., Witek, T.J., Jr.: A long-term evaluation of once-daily inhaled tiotropium in chronic obstructive pulmonary disease. Eur. Respir. J. 19(2), 217-224 (2002)

13. Vincken, W., van Noord, J.A., Greefhorst, A.P., Bantje, T.A., Kesten, S., Korducki, L., Cornelissen, P.J.: Improved health outcomes in patients with COPD during 1 year's treatment with tiotropium. Eur. Respir. J. 19(2), 209-216 (2002)

14. Brusasco, V., Hodder, R., Miravitlles, M., et al.: Health outcomes following treatment for six months with once daily tiotropium compared with twice daily salmeterol in patients with COPD. Thorax 58(5), 399-404 (2003)

15. Briggs, A.H., Sculpher, M.: An introduction to Markov modelling for economic evaluation. Pharmacoeconomics 13(4), 397-409 (1998)

16. Briggs, A.: Probabilistic analysis of cost-effectiveness models: statistical representation of parameter uncertainty. Value Health 8(1):1-2 (2005)

17. Oostenbrink, J.B., Rutten-van Mölken, M.P.M.H., Monz, B.U., FitzGerald, J.M.: Probabilistic Markov model to assess the cost-effectiveness of bronchodilator therapy in COPD patients in different countries. Value Health 8(1), 32-46 (2005)

18. Scanlon, P.D., Connett, J.E., Waller, L.A., Altose, M.D., Bailey, W.C., Buist, A.S.: Smoking cessation and lung function in mild-to-moderate chronic obstructive pulmonary disease. The Lung Health Study. Am. J. Respir. Crit. Care Med. 161, 381-390 (2000)

19. Miravitlles, M., Murio, C., Guerrero, T.: Factors associated with relapse after ambulatory treatment of acute exacerbations of chronic bronchitis. DAFNE Study Group. Eur. Respir. J. 17(5), 928-933 (2001)

20. Miravitlles, M., Ferrer, M., Pont, A., Zalacain, R., AlvarezSala, J.L., Masa, F., Verea, H., Murio, C., Ros, F., Vidal, R.: Effect of exacerbations on quality of life in patients with chronic obstructive pulmonary disease: a 2 year follow up study. Thorax 59(5), 387-395 (2004)

21. Decramer, M., Celli, B.R., Tashkin, D.P., Pauwels, R.A., Burkhart, D., Cassino, C., Kesten, S.: Clinical trial design considerations in assessing long-term functional impacts of tiotropium in COPD: the Uplift trial. J. Chron. Obstruct. Pulm. Dis. 1(2), 303-312 (2004)

22. Badia, X., Roset, M., Herdman, M., Kind, P.: A comparison of United Kingdom and Spanish general population time trade-off values for EQ-5D health states. Med. Decis. Making 21(1), 7-16 (2001)

23. Paterson, C., Langan, C.E., McKaig, G.A., Anderson, P.M., Maclaine, G.D., Rose, L.B., Walker, S.J., Campbell, M.J.: Assessing patient outcomes in acute exacerbations of chronic bronchitis: the measure your medical outcome profile (MYMOP), medical outcomes study 6-item general health survey (MOS-6A) and EuroQol (EQ-5D). Qual. Life Res. 9(5), 521-527 (2000)

24. Spencer, S., Jones, P.W.: Time course of recovery of health status following an infective exacerbation of chronic bronchitis. Thorax 58(7), 589-593 (2003)

25. Miravitlles, M., Murio, C., Guerrero, T., Gisbert, R.: Pharmacoeconomic evaluation of acute exacerbations of chronic bronchitis and COPD. Chest 121(5), 1449-1455 (2002)

26. Miravitlles, M., Murio, C., Guerrero, T., Gisbert, R.: Costs of chronic bronchitis and COPD: a 1-year follow-up study. Chest 123(3), 784-791 (2003)
27. Institute for Medical Statistics (IMS) Health: El Marcado Farmaceutico Spain. http://www.imsportal.com (restricted access). Accessed July 2005

28. Rovira, J., Antonanzas, F.: Economic analysis of health technologies and programmes. A Spanish proposal for methodological standardisation. Pharmacoeconomics 8(3), 245-252 (1995)

29. Briggs, A.H.: Handling uncertainty in cost-effectiveness models. Pharmacoeconomics 17(5), 479-500 (2000)

30. van Hout, B.A., Al, M.J., Gordon, G.S., Rutten, F.F.H.: Costs, effects and c/e-ratios alongside a clinical trial. Health Econ. 3, 309-319 (1994)

31. Briggs, A.H., Fenn, P.A.: Confidence intervals or surfaces? Uncertainty on the cost-effectiveness plane. Health Econ. 7, 723-740 (1998)

32. Fenwick, E., Claxton, K., Sculpher, M.: Representing uncertainty: the role of cost-effectiveness acceptability curves. Health Econ. 10(8), 779-787 (2001)

33. Stinnett, A.A., Mullahy, J.: Net health benefits: a new framework for the analysis of uncertainty in cost-effectiveness analysis. Med. Decis. Making 18(2 Suppl), S68-S80 (1998)

34. Esteban, C., Moraza, J., Aburto, M., Quintana, J.M., Capelastegui, A.: Description of a sample of patients with chronic obstructive pulmonary disease treated at hospitalsupervised respiratory clinics at primary care centers. Arch. Bronconeumol. 39(11), 485-490 (2003)

35. Sacristan, J.A., Oliva, J., Del Llano, J., Prieto, L., Pinto, J.L.: What is an efficient health technology in Spain? (in Spanish). Gac. Sanit. 16(4), 334-343 (2002)

36. Langley, P.C.: The NICE reference case requirement: implications for drug manufacturers and health systems. Pharmacoeconomics 22(4), 267-271 (2004)

37. Chapman, R.H., Stone, P.W., Sandberg, E.A., Bell, C., Neumann, P.J.: A comprehensive league table of cost-utility ratios and a sub-table of "panel-worthy" studies. Med. Decis. Making 20(4), 451-67 (2000)

38. Laupacis, A., Feeny, D., Detsky, A.S., Tugwell, P.X.: How attractive does a new technology have to be to warrant adoption and utilization? Tentative guidelines for using clinical and economic evaluations. Can. Med. Assoc. J. 146(4), 473-481 (1992)

39. Anzueto, A., Tashkin, D., Menjoge, S., Kesten, S.: One-year analysis of longitudinal changes in spirometry in patients with COPD receiving tiotropium, Pulm. Pharmacol. Ther. 18(2), 75-81 (2005)

40. Donaldson, G.C., Seemungal, T.A., Bhowmik, A., Wedzicha, J.A.: Relationship between exacerbation frequency and lung function decline in chronic obstructive pulmonary disease. Thorax 57(10), 847-852 (2002)

41. Kanner, R.E., Anthonisen, N.R., Connett, J.E.: Lower respiratory illnesses promote $\mathrm{FEV}(1)$ decline in current smokers but not ex-smokers with mild chronic obstructive pulmonary disease: results from the lung health study. Am. J. Respir. Crit. Care Med. 164(3), 358-364 (2001)

42. Sculpher, M.J., Buxton, M.J.: The episode-free day as a composite measure of effectiveness: an illustrative economic evaluation of formoterol versus salbutamol in asthma therapy. Pharmacoeconomics 4(5), 345-352 (1993)

43. Richter, A., Anton, S.E., Koch, P., Dennett, S.L.: The impact of reducing dose frequency on health outcomes. Clin. Ther. 25(8), 2307-2335 (2003) 